\title{
Relationship Between Beats Above Baseline Index (BABI) and Physiological Cost Index (PCI) During Walking with Leg Length Discrepancy
}

J. Phys. Ther. Sci.

8: $13-18,1996$

\author{
HITOSHI TAKEI ${ }^{1)}$ \\ 1) Department of Physical Therapy, Tokyo Metropolitan College of Allied Medical Sciences, \\ 2-10, 7-chome, Higashiogu, Arakawa-ku, Tokyo 116, Japan. TEL +81-3-3819-1211.
}

\begin{abstract}
The purpose of this study was to assess the criterion-referenced validity of the beats above baseline index (BABI) in relation to physiological cost index (PCI) by having subjects walk with leg length discrepancy and to evaluate the need to supplement shoe height to compensate for leg length discrepancy based on the changes in BABI and PCI in 8 healthy men. The exercise task in the experiments was continuous walking of a 200 -m distance along a figure " 8 "-shaped path, each circle of which was $30 \mathrm{~m}$ long. The degree of task difficulty varied among 5 different gaits with leg length discrepancies of $0 \mathrm{~cm}$ to $4 \mathrm{~cm}$ under two conditions: comfortable walking speed and maximal walking speed. The criterion-referenced validity of BABI in relation to PCI was high at $r=0.85-0.89$, demonstrating that BABI can be applied to walking tasks. There were no significant differences between time for task completion, walking speed, stride length or cadence at the two walking speeds when leg length discrepancy was no more than $4 \mathrm{~cm}$. At the comfortable walking speed, however, there were significant individual differences between BABI and PCI at differences in leg length of $4 \mathrm{~cm}$ and $0 \mathrm{~cm}$, of $4 \mathrm{~cm}$ and $1 \mathrm{~cm}$, and of $4 \mathrm{~cm}$ and $2 \mathrm{~cm}$. When gait withleg length discrepancies is considered from the standpoint of task difficulty assessed by BABI and energy expenditure assessed by PCI, supplementing shoe height is considered necessary at leg length differences of $4 \mathrm{~cm}$ or more
\end{abstract}

Key words: Beats above baseline index, Physiological cost index,Leg length discrepancy.

(This article was submitted Mar. 13, 1996, and was accepted May 10, 1996)

\section{INTRODUCTION}

Degree of task difficulty can be evaluated on the basis of metabolic equivalents (METs), heart rate (HR), blood pressure and respiratory rate, but all of them are evaluated based on only one variable. They tend to be influenced by the examinee's motivation and are seldom applied to functional evaluation. Parameters for the ability to move, on the other hand, rely on two variables, and include the physiological cost index (PCI) and beats above baseline index (BABI) advocated by Pine et al. ${ }^{1)}$ in 1994. Both parameters have in common the fact that HR is used for one of the two variables.
BABI is used to assess task difficulty, and uses effort (reflected in HR changes) and time for task completion as the variables. This index is mathematically based on the integral of effort (estimated by instantaneous HR increase) across the time interval for task completion. This can be visualized as the area under the curve for HR increase (Yaxis) versus task time (X-axis). BABI is being tested for reliability and validity at present ${ }^{1 / 2)}$, and its criterion-referenced validity has not yet been assessed in relation to any other index using two variables. BABI is calculated by computer processing of the actual integral values or by using a formula to estimate it. In our previous study ${ }^{3)}$, a strong positive correlation was found between 
BABI values obtained by computer processing and by the estimation formula with a Pearson's product moment correlation coefficient of 0.9998 . This is close to that $(r=0.95)$ in the previous study by Pine et al. ${ }^{1)}$. The intraclass correlation coefficient (ICC $[3,1]$ ) between the first test (test) and the second test (retest) was also high, 0.77-0.86, thus confirming, as in the previous study by Pine et al. ${ }^{1)}$, high test-retest reproducibility.

PCI ${ }^{4) 5}$ ), on the other hand, an index of walking efficiency, has been investigated for validity5), reliability $^{6)}$ and reproducibility ${ }^{7)}$ in many fields ${ }^{8-11)}$, and has been demonstrated to be useful. The fact that PCI is closely correlated with METs $(r=0.88)$ in particular suggests that this index can be useful in assessing energy expenditure ${ }^{6}$ ). In addition, the use of the figure " 8 "-shaped path has the advantage of allowing bilateral shifting of the center of gravity, as opposed to walking in a straight line or walking in circles in the same direction.

The purpose of this study was to assess the criterion-referenced validity of $\mathrm{BABI}$ in relation to PCI in healthy persons performing a walking task with the length of one leg of intentionally varied. The influence of gait at different leg lengths on BABI as an index of task difficulty, PCI as an index for walking efficiency, and various other factors were investigated in healthy subjects. The need to supplement shoe height was also assessed based on changes in these indices.

\section{METHODS}

\section{Subjects}

The subjects were 8 healthy men with no differences in leg length. Their mean age was 19.8 (18-22) years, mean height $171.8 \pm 5.7 \mathrm{~cm}$, mean body weight $66.1 \pm 4.5 \mathrm{~kg}$, and mean leg length (SMD) $83.8 \pm 2.9 \mathrm{~cm}$. The purpose of the experiment was explained to all of the subjects, and prior consent was obtained from each of them. The subjects were prohibited from drinking, eating and smoking for 2 hours before the start of the experiment.

\section{Procedures and study design}

Time for task completion and changes in HR were the variables for BABI, with distance and pace constant. The exercise task used in this study was walking, because the relation of BABI to PCI was going to be assessed. In the measurement of
PCI performed by MacGregor et al.4)5), examinees were asked to walk continuously along an indoor figure " 8 "-shaped path $(30 \mathrm{~m} /$ circle $)$ for 3 minutes or $200 \mathrm{~m}$. The present experiment used a continuous 200-m walking course, the distance was constant, and time was used as a variable.

The walking task included a total of 10 items. Differences in leg length of 1,2, 3 and $4 \mathrm{~cm}$ with 0 $\mathrm{cm}$ as the standard difference in leg length were used, with two speeds, "comfortable walking speed" and "maximal walking speed", which varied with the examinee for each of these 5 items. For comfortable walking speed, the examinees were asked to walk at their usual speed at which they walk most easily. For maximal walking speed, they were asked to walk as fast as they could. The leg length discrepancy was produced by attaching layers of hard rubber $(1 \mathrm{~cm}$ thick and weighing $80 \mathrm{~g}$ ) to the entire sole of the left shoe. The part of the sole distal to the site corresponding to the metatarsophalangeal (MP) joint was beveled to facilitate changing steps.

Measurements were first made in each subject with a difference in leg length of $0 \mathrm{~cm}$. The subsequent measurements for each item were performed at random on different days. There were rest intervals of 7 minutes or more between tasks to allow the HR to return to its resting value. The examinees were instructed to dress lightly and to wear Partner shoes (Ueyama Co., Ltd.) during the experiment. They were also asked to practice walking with the shoes for about 12 hours before the experiment to accustom themselves to wearing shoes that made their leg length different.

\section{Measures}

HR was measured at a memory interval of $5 \mathrm{sec}$ with a heart rate monitor VANTAGE XL (POLAR ELECTRO OY Co., Ltd., Finland). The examinees sat resting for 15 minutes, and the final 300 seconds was divided into ten 30 -second periods. The mean value for each 30 -sec period was determined. The lowest mean of the values for the 10 periods was regarded as baseline HR (bts/min) ${ }^{1}$. Exercise HR was calculated from the values measured during the 10 seconds immediately before the end of experiment. Walking speed was calculated from the 200-m walking distance and time to complete the walk. Stride length was calculated by dividing the distance walked per minute by the number of strides per minute. 
The items analyzed were BABI, difference between the mean HR during exercise and baseline HR (MBHR), time for task completion (TIMEtask), PCI, difference between HR during exercise and HR at rest (baseline HR) (WBHR), walking speed (SPEED), stride length, and cadence. BABI was calculated from the following estimation formula ${ }^{1)}$ :

$\mathrm{BABI}$ (beats) $=$

TIMEtask (sec) $\times$ MBHR (beats/min) $\div 60(\mathrm{sec})$

PCI was calculated from the following formula ${ }^{4) 5)}$ :

PCI (beats $/$ meter $)=$ WBHR $($ beats $/ \mathrm{min}) \div$ SPEED $(\mathrm{m} / \mathrm{min})$

\section{Analysis}

Means and standard deviation (SD) for each item analyzed were calculated. Task difficulty for each item was assessed by analysis of variance and a multiple comparison test (LSD). Pearson's product moment correlation coefficients for each of the two speeds and BABI were calculated to assess criterion-referenced validity in relation to PCI. Pearson's product moment correlation coefficients for BABI, MBHR and TIMEtask, and for PCI, WBHR and SPEED were calculated to assess the influence of MBHR and TIMEtask on BABI, and of WBHR and SPEED on PCI. Statistical analysis was performed with SPSS/PC+.

\section{RESULTS}

Table 1 shows the means and SD of items analyzed at each of the differences in leg length and the results of the assessment by LSD. BABI and its relation to MBHR and TIMEtask, which influence BABI, were investigated at each difference in leg length. BABI and MBHR tended to increase as the leg length discrepancy increased at comfortable walking speed, and both parameters also showed a tendency to increase at maximal walking speed, but only slightly. TIMEtask remained approximately constant regardless of leg length discrepancy at both walking speeds. The aforementioned tendency was marked at the maximal walking speed. Two-way analysis of variance of examinee factors and the leg length discrepancy factor revealed significant $(\mathrm{p}<0.01)$ differences between BABI and MBHR values at different leg lengths at the comfortable walking speed. Assessment of these items by LSD revealed significant differences between BABI values at leg length differences of $4 \mathrm{~cm}$ and $0 \mathrm{~cm}, 4 \mathrm{~cm}$ and $1 \mathrm{~cm}$, and 4 $\mathrm{cm}$ and $2 \mathrm{~cm}$. Similar results were obtained for MBHR. The assessment of PCI and variables that influence it yielded data showing that PCI is correlated with BABI, WBHR with MBHR, and SPEED with TIMEtask. There were no significant differences in stride length or cadence.

Table 2 shows Pearson's product moment correlation coefficients for each walking speed and $\mathrm{BABI}$ to assess the criterion-referenced validity in relation to PCI. There was a very close correlation between $\mathrm{BABI}$ and PCI, and there were also close correlations between MBHR and BABI and between WBHR and PCI.

\section{DISCUSSION}

\section{Criterion-referenced validity of $B A B I$ in relation to $P C I$}

TIMEtask was approximately constant for BABI in the present task, and SPEED was approximately constant for PCI. These findings show that both BABI and PCI have been influenced by changes in HR. The results of LSD and the Pearson's product moment correlations show that the criterion-referenced validity of BABI in relation to PCI is high. If TIMEtask increased as the difficulty of the present task increased, SPEED should decrease. Here too, both BABI and PCI would be expected to increase and the correlation between the two indices should be close. Thus, BABI is considered to have high criterion-referenced validity in relation to PCI, and to be possible index for assessing the difficulty of the present task. The discussions in the following sections are based on these findings.

\section{2. $B A B I$}

Pine et al. ${ }^{1)}$ prepared two grades of task difficulty in the task of climbing 6 floors with each of the staircases consisting of 11 stairs, with normal stair climbing as a control, in order to investigate the reliability and validity of BABI. One grade of task difficulty consisted of climbing stairs with a cane in the right hand, with the left hand on the handrail and a device to maintain the knee of the left leg extended. The other grade consisted of climbing stairs on a pair of crutches without loading the left leg. When healthy adults performed these tasks of climbing, BABI increased signifi- 
Table 1. Means (upper column) and SD (lower column) for each item measured and results of LSD

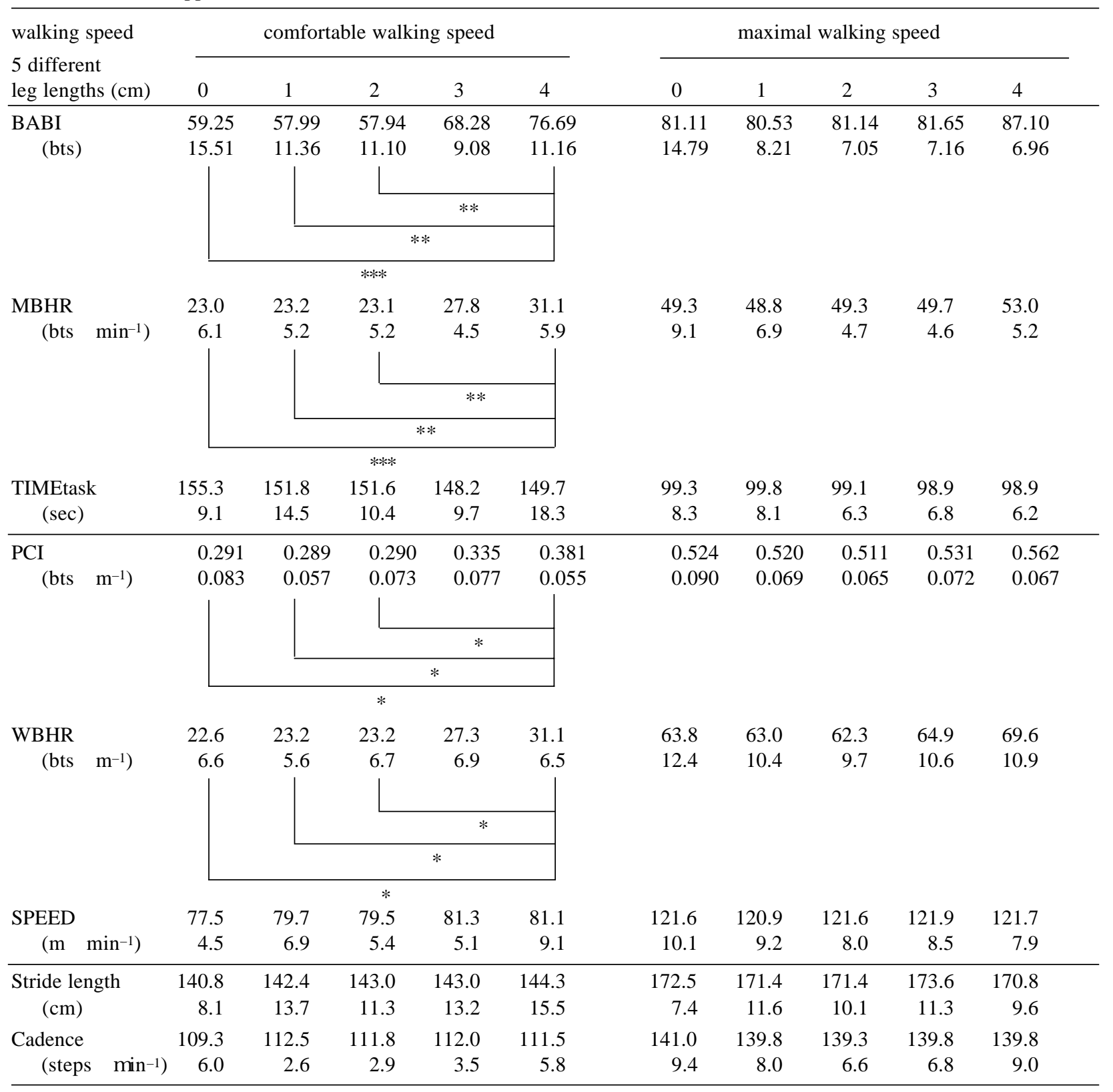

$* * * \mathrm{p}<0.005, * * \mathrm{p}<0.01, * \mathrm{p}<0.05$.

cantly, and TIMEtask also increased significantly. However, the difference between maximal HR during exercise and baseline HR on the second task (with the former grade of difficulty) was less than during normal stair climbing and on the third task (with the latter grade of difficulty). The results revealed that the range of motion of the knee joint on the second task increased task difficulty and that the second task requires a great deal of time despite the small range of changes in HR. As a result, Pine et al. concluded that BABI can be used to determine task difficulty and that, if task conditions are modified, the ability to move could be evaluated even in limited space. Further studies are, however, needed on the application of task fixation and the correlation between criterion-referenced validity and oxygen consumption.

In the present experiment, BABI was found to 
Table 2. Pearson's product moment correlation coefficient

\begin{tabular}{llll}
\hline & & PCI & \\
BABI & Comfortable & $0.890^{* *}$ & \\
\hline \multirow{6}{*}{ Maximal } & $0.847^{* *}$ & \\
& & MBHR & TIMEtask \\
\hline \multirow{2}{*}{ BABI } & Comfortable & $0.946^{* *}$ & -0.197 \\
& Maximal & $0.844^{* *}$ & 0.033 \\
& WBHR & SPEED & \\
PCI & Comfortable & $0.962^{* *}$ & 0.330 \\
& Maximal & $0.916^{* *}$ & 0.266 \\
\hline
\end{tabular}

$\mathrm{N}$ of cases: 40. 1-tailed signif: $*=0.01, * *=0.001$.

be closely correlated with HR, showing that TIMEtask was maintained approximately constant. If BABI reflected task difficulty, task difficulty should have increased at differences in leg length of $4 \mathrm{~cm}$ or more. TIMEtask remained approximately constant, probably because only leg length discrepancy was used to determine task difficulty. If the range of motion of the knee joint had been restricted, TIMEtask might also have increased. The results of the present experiment suggest that MBHR, which was particularly closely correlated with BABI, reflects the patient's effort stated by Pine et al.

\section{3. $P C I$}

The factors that directly influence PCI are WBHR and SPEED. In the present experiment, WBHR showed changes similar to those in PCI at approximately constant SPEED, suggesting that PCI had been influenced by changes in HR. The changes were particularly marked at the comfortable walking speed, suggesting that the elevated HR, which influenced PCI, is attributable to energy expenditure to raise the center of gravity upward after it had been lowered. There were no significant differences between stride length or cadence at the various differences in leg length. This finding supports the results of our previous study ${ }^{12}$ ) and of the study by Ebata et al. ${ }^{13)}$. When these various studies are taken into consideration, although the experimental conditions were different, walking speed appears to have been regulated at a constant rate in the determination of changes in the right stride width to the left stride width ratio. Walking practice for about 12 hours may also have been responsible for each examinee's practiced walking.

PCI is regarded as a parameter of walking efficiency, suggesting that supplementing shoe height is necessary for energy expenditure at a difference in leg length of $4 \mathrm{~cm}$. There were no significant differences between PCI values at the various differences in leg length at maximal walking speed, probably because the rate of energy expenditure for up-down movement was less than for anterior movement of the center of gravity for the examinee to maintain maximal speed, and the influence of leg length discrepancy was eventually lost. These findings suggest that the influence of leg length discrepancy at maximal walking speed is less than at comfortable walking speed. The BABI data suggest the same conclusion.

\section{Need to supplement shoe height to compensate for leg length discrepancy}

The above results and discussion suggest that individuals have the ability to become accustomed to walking in terms of TIMEtask, SPEED, stride length and cadence by randomly controlling the left to right stride width ratio, hip, knee and ankle joint angles, pelvic tilt and rotation angles, and shifting of the center of gravity at leg length differences of $4 \mathrm{~cm}$ or less. However, there were significant differences at the difference in leg length of $4 \mathrm{~cm}$, despite the fact that the task was completed after the individuals had to some degree become accustomed to walking with legs of different length. This suggests that a leg length discrepancy of $4 \mathrm{~cm}$ is the point at which effective compensation becomes difficult for energy expenditure even in healthy subjects. Therefore, when gait with different leg lengths is considered from the standpoint of BABI as an index of task difficulty and PCI as an index of energy expenditure, supplementing shoe height appears necessary for a leg length discrepancy of $4 \mathrm{~cm}$ or more.

\section{CONCLUSION}

BABI indicates low task difficulty when changes in HR are slight and TIMEtask is reduced. On the other hand, PCI indicates good efficiency when changes in HR are slight and SPEED increases. The results of the present study show that the criterion-referenced validity of BABI in relation to PCI is high and that both indices evaluate ability to walk from the same standpoints. In the present 
experiment, however, walking was chosen as the task to assess BABI in relation to PCI. BABI is not just a measurement procedure for walking, but BABI can be applied to a variety of tasks. Further studies are needed to determine which tasks BABI is reliable for and whether PCI can be substituted for BABI. In practice, however, $\mathrm{HR}$ at the end of walking is used for PCI, while all changes in HR during performance of the task must be monitored for BABI. In this respect, PCI may be simpler than BABI.

The results of the present experiment suggest that supplementing shoe height becomes necessary for leg length differences of $4 \mathrm{~cm}$ or more, when gait with differences in leg lengths is considered from the standpoint of BABI as an index of task difficulty and PCI as an index of energy expenditure.

\section{Acknowledgement}

The authors are indebted to the staff and students of our department for their kind cooperation.

\section{REFERENCES}

1) Pine ZM, Colbran E, Corpolongo R: Reliability and validity of the beats above baseline index; a new measure of task difficulty. Arch Phys Med Rehabil 75: 545-550, 1994.

2) Pine ZM, Teitelbaum S: Reliability and validity of the beats above baseline index, a new measure of task difficulty, applied to walking tasks in elderly subjects. Am J Phys Med Rehabil 72: 233, 1993 (abstract).

3) Takei H, Iwasaki K, et al: Task Difficulty of Going and Down the Step. 31th Meeting of Japan Physical Therapist, Rigaku Ryohogaku 23: 39, 1996 (abstract).

4) MacGregor J: Rehabilitation ambulatory monitoring. In: Kenedi RM, Paul JP, Hughes J (eds), Disability. Macmillan, London, 1979, pp 159-172.

5) MacGregor J: The evaluation of patient performance using long-term ambulatory monitoring technique in the domiciliary environment. Physiotherapy 67: 3033, 1981.

6) Takei H, Iwasaki K: Reliability of PCI as an index for energy expenditure: in comparison with energy expenditure in $\mathrm{mlO}_{2} / \mathrm{m} \bullet \mathrm{kg}$ as to the optimal walking speed. J Phys Ther Sci 5: 19-26, 1993.

7) Koseki T, Yamamoto S, et al: Reliability of physiological cost index-Difference of measurements for five consecutive days-.Tohoku Rigaku-Ryohogaku 3: 26-29, 1991.

8) Takei H, Iwasaki K, et al: Relationship between mode of walking and Physiological Cost Index. Undo Ryoho Kenkyukai Koen Ronbunshu 17: 30-33, 1992.

9) Wada I, Ikeda T, et al: The energy consumption and cardiac tasks during free level walking in preoperative patients with osteoarthritic hip. Rihabiriteshon igaku (Jap J Rehabil Med) 30: 49-53, 1993.

10) Nene AV: Physiological cost index of walking in able-bodied adolescents and adults. Clin Rehabil 7: 319-326, 1993.

11) Shimizu C, Kameda M, et al: The relationship of PCI to ambulatory speed in healthy adult females. Rigaku Ryohogaku 22: 449-453, 1995.

12) Takei H, Iwasaki K, et al: Effect of difference in leg length on walking. J Exerc Physiol 6: 193-199, 1991.

13) Ebata K, Satoh K, et al: Influence of leg length discrepancy on gait. J of Joint Surgery 11: 17-25, 1992. 